\title{
Dynamic simulation of the effect of vehicle-side pressure loss of hydrogen fueling process
}

Rothuizen, E.; Elmegaard, B.; Rokni, M.

Published in:

International Journal of Hydrogen Energy

Link to article, DOI:

10.1016/j.ijhydene.2020.01.071

Publication date:

2020

Document Version

Peer reviewed version

Link back to DTU Orbit

Citation (APA):

Rothuizen, E., Elmegaard, B., \& Rokni, M. (2020). Dynamic simulation of the effect of vehicle-side pressure loss of hydrogen fueling process. International Journal of Hydrogen Energy, 45(15), 9025-9038.

https://doi.org/10.1016/j.ijhydene.2020.01.071

\section{General rights}

Copyright and moral rights for the publications made accessible in the public portal are retained by the authors and/or other copyright owners and it is a condition of accessing publications that users recognise and abide by the legal requirements associated with these rights.

- Users may download and print one copy of any publication from the public portal for the purpose of private study or research.

- You may not further distribute the material or use it for any profit-making activity or commercial gain

- You may freely distribute the URL identifying the publication in the public portal 


\title{
Dynamic simulation of the Effect of vehicle-side pressure loss of hydrogen fueling process
}

\author{
E. Rothuizen ${ }^{\mathrm{a}, *}$, B.Elmegaard ${ }^{\mathrm{a}}$, M.Rokni ${ }^{\mathrm{a}}$ \\ ${ }^{a}$ Section for Thermal Energy Systems, The Technical University of Denmark, \\ 2800 Kgs. Lyngby, Denmark
}

\begin{abstract}
This study explains the fundamental mathematical equations used for the main component models that are implemented in freely available library for hydrogen fuelling station. The paper provides a background to the model formulation and theory, useful for the further investigations of hydrogen fuelling stations. The model was verified against a specific manufacturer model, and it was validated by using test data from an actual fuelling station. The study works as documentation and validation of the model formulation. The simulation library is used to make a model for investigating how the the pressure loss in the vehicle affects the fuelling process. Keeping the temperature out of the station constant and fuelling to $80 \mathrm{MPa}$ in the compressed hydrogen storage system, the pressure loss in the compressed hydrogen storage system directly correlates to the final temperature. The final temperature increases with increasing pressure losses. It is also shown that with no pressure loss in the vehicle the fuelling has no limit in fuelling speed as the heat of compression depends on the mass filled and the enthalpy of the mass, and not the filling time.
\end{abstract}

Keywords: Hydrogen fuelling, Temperature development, Pressure loss, Optimization, Dynamic simulation model

\section{Introduction}

The first hydrogen fueling stations installed for vehicles for personal transportation dates back to year 2000 and forward. The first protocol for fuelling personal vehicles, SAE TIR J2601, was developed by Society of automotive engineers and released in 2010 [26]. The protocol defined static pressure ramp rates for fuelling depending on several parameters such as ambient temperature, starting pressure in vehicle, communication etc.. The protocol SAE TIR J2601 was extended with more tables and developed to a standard in 2014 [27]. In 2016 the MC fuelling method was added to the standard [28]. The MC fuelling method is dynamic, using real time calculations during fueling to adjust the ramp rate, reducing fuelling time without compromising safety.

Even though the hydrogen fuelling technology is relatively new, many research projects has already been conducted. In general the research within the thermodynamics of hydrogen fueling can be divided into two categories; system considerations and component consid-

\footnotetext{
${ }^{*}$ Corresponding author, Tel.: +45 4525 4329, Fax: +45 45884325 Email address: edro@mek.dtu.dk (E. Rothuizen)
}

eration where the storage tank is by far the most dominating. Considering the system simulations the analyses have been conducted from a broader system perspective with Maus et al. [16] being the first to use and describe the protocol. Farzaneh-Gord et al. [8] compared a cascade fuelling with a single tank fuelling and showed that the cascade system was more energy efficient but slower than the single tank filling. Kuroki et al. [30] considers a fuelling from the break away to the FCE storage. Dynamic simulations of complete hydrogen fueling stations has been conducted by Schäfer et al. [31] who considers a fueling station with cryopump technology and proposes a new method of controlling the hydrogen temperature by mixing two hydrogen mass flow with different temperature. A dynamic model considering the whole process fuelling the station and the vehicle was developed by Reddi et al. [21], the model was used to investigate several operation strategies and comparing the SAE J2601 lookup table based fueling with the MC method for fueling [22]. The thermodynamics of filling a hydrogen tank has been investigated by exergy analysis, and it has been shown that increasing the initial pressure increases the exergy efficiency and results in a lower final temperature in the hydrogen tank [11]. 
Similar work was conducted by Ozsaban et al. [20].

Recent studies are expanded to consider back to back fuelings. The cost of the refrigeration system depending on the allowed temperature rise and numbers of back to back fuelings have been investigated by Elgowainy et al. [7].

Considering the studies of the vehicles compressed hydrogen storage system. Some of the first comprehensive studies on compressed hydrogen fueling were carried out by Dicken and Merida [5]. The study showed a non-uniform temperature distribution in the tank with up to a $6{ }^{\circ} \mathrm{C}$ temperature difference. Further, the study showed that the main contributor to the heat generation in the tank was the compression rather than the JouleThomson effect, this was also shown by Striednig et al. [33]. Although the study of Dicken and Merida [5] showed a non-uniform temperature distribution during the fueling, it is generally accepted that in mathematical models the temperature in the tank can be assumed uniform, according to the research conducted by Monde et al. [19, 18] and Woodfield et al. [34]. Further they showed that new tanks have a distributor at the inlet to ensure a more uniform temperature distribution. Monde et al. [19] and Woodfield et al. [35] have made different models of both analytical and numerical transient heat transfer from the hydrogen through the tank wall and to the ambient. The average local hydrogen heat transfer coefficient during charging vessels was estimated. For discharging vessels, Daney's relation has been shown to be valid for a large range of Rayleigh numbers [35], further studies has shown that the dominant heat transfer mode is natural convection and that the center temperature of the vessel i representative when determining heat transfer number [29]. Galassi et al. [9] conducted experiments on the life-time of the tanks, and the local temperature inside the tank was measured and compared to the outside temperature. The results were used to validate a CFD model of the tank during fast filling. Similar work has been carried out by Heitsch et al. [10] and Abhilash. [1]. The role of the initial tank temperature has been analysed with respect to the final temperature after the filling, it showed a linear correlation between the two temperatures [17]. Bourgeois et al. [4] has done a comprehensive study on hydrogen vessels conducting experiments to measure the heat transfer number inside type 3 and 4 tanks for different mass flow rates and nozzle diameters. The study suggest a correlation for Nusselts number based on their test data. Furthermore, the study compare their measured end of fill tamperatures to the calculated with good consistency and the study also considers stratification using CFD modelling.

$\mathrm{Li}$ et al. [15] has done investigations on the tempera- ture rise inside the hydrogen tank. It was found that the lowest temperature increase was obtained when filling with linearly decreasing mass flow rate.

Farzaneh-Gord et al. [8] has done a comparison between cascade fueling and fueling from a single tank. The results revealed that the filling time of the single tank was faster than for the cascade system but, it was also accompanied with a much higher entropy generation. The study also revealed that it required less work for the compressor for the cascade system.

Zhao et al. [37] has conducted research using numerical simulations to predict the temperature rise in the hydrogen tank during filling. The results showed that with increased mass flow rates, the temperature would increase, and with an increasing initial pressure the temperature would also decrease. The fueling time constraints due to the thermodynamics in the tank has been investigated by Yang . [36]. The fueling time is compared to the upper and lower limits of the tank properties for both adiabatic and isothermal conditions. The fueling time was shortest under adiabatic conditions.

This paper aims to fill the gap of thermodynamic modelling of hydrogen fueling stations considering all relevant component from the storage system in the fueling station to the vehicle storage system. The modelling describes all the processes which take place from the on-site storage at the fueling station to the onboard storage in the vehicle, including but not limited to heat transfer in the tanks and the tubes of different materials, the different temperature changes caused by pressure losses and heat exchange, the compression of hydrogen in a compressor and inside the vessels. The model formulation is based on real gas behaviour. Rothuizen et al. [23, 24] previously described a simpler thermodynamic model of a hydrogen fueling station, which was limited to analyse the thermodynamics of a cascade fueling station. This paper continues the former work by further development of the model including; different kinds of pressure losses, allowing zero mass flow and flow in both directions of a component, new compressor characteristics, a user interface with drag and drop components and updated fluid property library for increased calculation speed. These changes enables the possibility to model various hydrogen fueling station designs. Furthermore, a verification and a validation of the model is described and carried out in this work. The methods presented in this paper were implemented in the open source Modelica library for modelling hydrogen fueling stations by Rothuizen [38]. 


\section{Methods}

This section describes the complete model formulation and the mathematical formulation used for making a generic dynamic system model of hydrogen fueling. For each component model there is a short introduction followed by general information and properties of the component and then the model description. The methods provide a basic understanding of the volumes, heat losses, pressure losses, the compressor and heat exchanger. Furthermore, the model is verified and validated against a third party model and actual test data from a hydrogen fueling station. An extended description of the methods can be found in the Ph.D. thesis "Hydrogen Fueling Stations - Thermodynamic Analysis of Fuelling Hydrogen Vehicles for Personal Transportation" of Rothuizen [25].

\subsection{First principles}

The component models used for the simulation of hydrogen fulling systems are based on the first law of thermodynamics, Newton's second law for impulse and mass conservation. These are used for energy balance, momentum balance and mass balance, respectively. The first law of thermodynamics is stated in eq. 1 .

$$
\begin{array}{r}
\frac{d E}{d t}=\dot{Q}-\dot{W} \\
+\sum\left(\dot{m}\left(h+\frac{V^{2}}{2}+g z\right)\right)_{\text {in }} \\
-\sum\left(\dot{m}\left(h+\frac{V^{2}}{2}+g z\right)\right)_{\text {out }}
\end{array}
$$

For all the components it has been assumed that the gravitational potential energy may be neglected, and the kinetic energy is simplified through compressible gas flow relations. The kinetic energy is included in the enthalpy as the specific stagnation enthalpy has been used throughout the system; see Section 2.2 for further explanation. This leaves us with the energy balance for an open system, where the specific enthalpy is the stagnation enthalpy.

$$
\frac{d U}{d t}=\dot{Q}-\dot{W}+\sum(\dot{m} h)_{\text {in }}-\sum(\dot{m} h)_{\text {out }}
$$

For steady state which is used in the quasistationary control volumes, the change of energy content is zero, hence $d E / d t=0$.

Newton's second law states that the change of momentum can be expressed through change in pressure as shown in eq. 3 .

$$
\sum F=A_{1} p_{1}-A_{2} p_{2}
$$

where $A_{1}=A_{2}$ and knowing that $p=F / A$, the momentum balance yields a pressure difference.

$$
\Delta p=p_{1}-p_{2}
$$

The mass balance states that the change in mass for an open system is

$$
\frac{d m}{d t}=\sum \dot{m}_{\text {in }}-\sum \dot{m}_{\text {out }}
$$

In general eqs. 2, 4 and 5 are present for all the models. However, they are formulated according to the principles of the operation of each component and omit irrelevant terms.

\subsection{Compressible gas flow relations}

The compressible gas flow relation considers stagnation (or total) enthalpy, static enthalpy and dynamic enthalpy. The stagnation enthalpy $(h)$ is the static enthalpy of the stream $\left(h_{s}\right)$ added to the dynamic part representing kinetic energy of the stream [6] .

$$
h=h_{s}+\frac{1}{2} v^{2}
$$

The kinetic energy is accordingly included in the stagnation enthalpy. This paper assumes that all enthalpies are stagnation enthalpies. Using stagnation enthalpy in a system with mass flow between components, the thermodynamic properties might deviate as the kinetic energy is included in the enthalpy used to find the thermodynamic properties. Though in this paper the kinetic energy never composes more than $2 \%$ of the stagnation enthalpy during a hydrogen fueling.

\subsection{Tank}

This section covers the modelling of the tanks with first an introduction with some general information about hydrogen tanks and then an explanation of the modelling theory.

\subsubsection{Tank types for storing hydrogen}

There are four different types of tanks for storing hydrogen; they are categorized as Types I to Type IV. As a general rule, the higher the type number, the higher pressure it is capable of storing and the more expensive the tank will be. Type I is made of stainless steel and can typically handle 200 bar to 350 bar pressure, but it has been made for pressures up to 500 bar. Type II is made from aluminium, it is lighter than Type I and can handle the same pressures. Type III and type IV are composite tanks, the former with a thin aluminium liner and the latter with a plastic liner wrapped in carbon fibre. They are lighter than Type I and Type II and can store hydrogen at 350 bar to more than a 1000 bar. 


\subsubsection{Tank model}

The tank model is based on the first principles with momentum blance, mass balance and energy balance. The momentum balance is $p=p_{1}=p_{2}$ so that the pressure into the tank is the same as in the tank; hence, there is no pressure loss at the entrance. The thermal boundary condition for the tank is defined by the heat exchange with the surroundings. The mass balance for the control volume is

$$
\frac{d M}{d t}=\dot{m}_{1}+\dot{m}_{2}
$$

Introducing $M=V \rho$ and assuming a constant volume of the tank, eq. 7]can be expressed as

$$
V \frac{d \rho}{d t}=\dot{m}_{1}-\dot{m}_{2}
$$

The thermodynamics of the tank can be stated from the first law of thermodynamics for an open system without any work added. The energy balance for one tank is the change in internal energy as mass leaves or enters the tank and the heat dissipation through the tank wall. The change in internal energy can be found from eq. 9

$$
\frac{d U}{d t}=\dot{m}_{1} h_{1}+\dot{m}_{2} h_{2}+\dot{Q}
$$

where $d U / d t$ is the change in internal energy of the system, $h_{1}$ and $h_{2}$ are the enthalpy leaving or entering the tank at each opening, $\dot{m}$ is the mass flow rate and $\dot{Q}$ is the heat rate entering or leaving the system, where entering is considered positive. Rewriting eq. 9 using $d U=d(M u)=V d(\rho u)$ gives

$$
V \frac{d(\rho u)}{d t}=\dot{m}_{1} h_{1}+\dot{m}_{2} h_{2}+\dot{Q}
$$

Rewriting and substituting internal energy with enthalpy $u=h-p v$ into eq. 11 gives

$$
\frac{d h}{d t}=\frac{1}{M} \cdot\left(\dot{m}_{1} h_{1}+\dot{m}_{2} h_{2}+V \frac{d p}{d t}+\dot{Q}\right)
$$

which gives the energy inside the tank expressed through enthalpy instead of internal energy. This rewriting is done as a results of the way the model is built up, to enable reverse mass flow from the tank.

\subsection{Heat transfer model}

The main heat transfer formulation used in the model is conduction through a wall. This formulation is valid for both the tanks which store the hydrogen and for the tubes in which the hydrogen flows. For both the tanks and the tubes the heat transfer is unsteady. In the tanks the temperature changes due to the heat of compression or expansion as the hydrogen flows to or from the tanks. For the tubes the hydrogen changes temperature as it flows through the tube. The second observation concerns whether or not a lumped model or a 1-dimensional model of the heat conduction should be used. Studying the tanks used for high-pressure hydrogen storage, one sees they are made of composite material with low heat conduction properties and that the walls are thick. From experiments at Saga University, the thermal conductivity of the liner and carbon fibre wrapping of the tanks have been measured and it shows that a 1-dimensional model is necessary [19]. For the tube the Biot number was calculated, and it showed that a lumped model is insufficient. Therefore a 1-dimensional model is formulated for both the tank and the tube model.

\subsubsection{1-dimensional unsteady heat transfer}

The heat transfer through the tank wall is assumed to be 1-dimensional unsteady heat conduction (the temperature of the gas changes when gas is leaving or entering the tank). The general heat equation and the boundary conditions of heat exchange through the wall are given in eqs. 12,13 and 14

$$
\begin{gathered}
\frac{d^{2} T_{s}}{\partial x^{2}}=\frac{1}{\alpha} \frac{\partial T_{s}}{d t} \\
\left.k \frac{d T_{w}}{d x}\right|_{x=0}=\alpha_{g}\left(T_{g}-\left.T_{w}\right|_{x=0}\right) \\
\left.k \frac{d T_{w}}{d x}\right|_{x=L}=\alpha_{a}\left(\left.T_{w}\right|_{x=L}-T_{a}\right)
\end{gathered}
$$

where $T_{w}$ is the wall temperature, $T_{g}$ the gas temperature in the tank and $T_{a}$ the air temperature outside the tank. $k$ is the thermal conductivity, $L$ the thickness of the wall, and $\alpha_{g}$ and $\alpha_{a}$ are the heat transfer coefficients of the gas in the tank and the air outside the tank, respectively. A numerical solution to eq. 12 can be obtained with a capacitance resistance method which corresponds to a finite volume analysis [13]. A node system can be defined with $i$ as the subscript identifying a node where the heat transfer occurs. Assuming a uniform temperature distribution of the gas at the inside of the wall and a wall acting like a plain wall, the resistance capacitance method can be applied to the hydrogen tank using eqs. 15 and 16

$$
Q_{A, i}=\sum_{j} Q_{k, i-j}=\left(Q_{k}\right)_{i, i-1}+\left(Q_{k}\right)_{i, i+1}
$$


and

$$
Q_{A, i}=(\rho c \Delta V)_{i} \frac{d T_{i}}{d t}+\dot{s}_{i} \Delta V_{i}
$$

where the subscript $i$ is the node where the temperature is calculated, and $\dot{s}_{i}$ is the rate of surface energy conversion. The resistance capacitance method simplifies the partial differential eq. 12 into an ordinary differential equation, eq. 16 The heat transfer $\left(Q_{k}\right)_{i \text { to } i-1}$ and $\left(Q_{k}\right)_{i \text { to } i+1}$ can be found from eqs. 17 and 18

$$
\begin{aligned}
Q_{k, i-j} & =\frac{T_{i}-T_{i-1}}{R_{i, i-1}} \\
Q_{k, i+j} & =\frac{T_{i}-T_{i+1}}{R_{i, i+1}}
\end{aligned}
$$

where $R$ is the thermal resistance of the volume, $R=$ $d x /(A k)$. The boundary conditions for eqs. 15 and 16 are given by eqs. 19 and 20 .

$$
\begin{aligned}
& \left.\frac{d T_{i}}{d x}\right|_{x=0}=\frac{\alpha_{g}}{k}\left(T_{g}-\left.T_{i}\right|_{x=0}\right) \\
& \left.\frac{d T_{i}}{d x}\right|_{x=L}=\frac{\alpha_{a}}{k}\left(\left.T_{i}\right|_{x=L}-T_{a}\right)
\end{aligned}
$$

Equations 17 and 18 are linked to the temperature change in time through eq. 21 .

$$
A \rho d x \frac{d T_{i}}{d t}=Q_{k, i-j}+Q_{k, i+j}
$$

where $A$ is the area of the tank, $d x$ the thickness of the tank layer considered, $\rho$ the density of the material and $d T_{i} / d t$ the change of temperature over time at the point.

\section{Tank}

The heat transfer coefficient depends on the tank design, the mass flow rate into the tank and the nozzle at the inlet. The heat transfer coefficient for a specific tank design may be estimated using CFD software. For deciding the heat transfer coefficient in the tank, several experiments have been carried out and they show that the heat transfer coefficient $\alpha_{g}$ varies between 150 $\mathrm{W} /\left(\mathrm{m}^{2} \mathrm{~K}\right)$ and $500 \mathrm{~W} /\left(\mathrm{m}^{2} \mathrm{~K}\right)$ [19], [35]. For emptying a tank, experiments have shown that Daney's correlation gives an acceptable approximate value [35]. The Nusselt number for Daney's correlation is given by eq. 22 [3].

$$
\mathrm{Nu}=0.104 \mathrm{Ra}^{0.352}
$$

where the Rayleigh number $(\mathrm{Ra})$ is given by eq. 23

$$
\mathrm{Ra}=\frac{g \beta\left(T_{w}-T_{g}\right) d^{3}}{v a}
$$

where $g$ is the gravitational acceleration, $\beta$ is the thermal expansion coefficient, $d$ is the inside diameter of the tank, $v$ is the dynamic viscosity of the gas and $a$ the thermal diffusivity which is $a=k /\left(\rho c_{p}\right)$, the thermal conductivity $(k)$ divided by the density $(\rho)$ and the specific heat capacity $c_{p}$. The outer tank wall is in contact with the ambient environment, and the heat transfer coefficient for the outside surface of the tank depends on different parameters, such as air flow, direction of the cylinder and if it is free or forced convection. The tanks are typically stored inside or on top of the station, where natural convection is common on the outside of the tank. The heat transfer coefficient is thus typically between $5 \mathrm{~W} /\left(\mathrm{m}^{2} \mathrm{~K}\right)$ and $30 \mathrm{~W} /\left(\mathrm{m}^{2} \mathrm{~K}\right)$ depending on the conditions.

\section{Tubes}

Hydrogen flowing through the tubes conducts heat through the tube wall. The heat transfer between the hydrogen and the wall is forced convection as the flow is turbulent. The heat transfer coefficients is found from the following set of equations [12].

$$
\alpha=\frac{\mathrm{Nu} \cdot k}{d_{h}}
$$

$$
\begin{gathered}
\mathrm{Nu}=0.023 \operatorname{Re}^{4 / 5} \operatorname{Pr}^{0.3} \\
\operatorname{Pr}=\frac{c_{p} \mu}{k} \\
\operatorname{Re}=\rho \frac{\dot{V}}{A_{\text {cross }}} \frac{d_{h}}{\mu}
\end{gathered}
$$

where $\alpha$ is the local heat transfer coefficient, $\mathrm{Nu}$ is Nusselt number, $\mathrm{Pr}$ is the Prandtl number which is a property parameter and Re is Reynolds number. $d_{h}$ is the hydraulic diameter, $k$ is the thermal conductivity, $c_{p}$ the specific heat, $\mu$ the viscosity, $\dot{V}$ the volume flow, $\rho$ the density and $A_{\text {cross }}$ the cross-sectional area of the tube. The heat transfer coefficient for the outside of the tube may be approximated using the same value as for the outside of the tank.

\subsection{Pressure losses}

This section covers the different components which cause pressure losses when hydrogen flows through. The pressure losses considered are either general pressure loss equations or equations given by manufacturers. Pressure losses in hydrogen fueling stations are not different from pressure losses of any other gas flow system. The components used are the same as for other high-pressure systems. 


\subsubsection{General pressure loss model}

There are three different pressure loss components which have the same energy, mass and momentum balance, but the final equation for calculating the pressure loss is different.

With reference to eq. 7 there is no work added $(W=0)$ and the process is adiabatic $(Q=0)$ in the pressure loss models. The mass entering the component must be the same as the mass leaving $\left(\dot{m}_{1}+\dot{m}_{2}=0\right)$ and the enthalpy is constant $\left(h_{1}=h_{2}\right)$. The momentum balance yields a pressure difference $\Delta p=p_{1}-p_{2}$, where $\Delta p$ is the pressure loss for each component. The changes when hydrogen undergoes a negative change in pressure are the pressure and the properties of the hydrogen. As the enthalpy is constant across the components and the pressure changes, the hydrogen properties (temperature, density, pressure, etc.) change. When hydrogen is throttled, the temperature increases due to the Joule-Thomson effect, which has a negative coefficient for hydrogen. Unlike most gases, the temperature of hydrogen increases when throttled at temperatures higher than $200 \mathrm{~K}$. The difference in temperature is approximately $0.04{ }^{\circ} \mathrm{C}$ per 1 bar pressure drop.

\subsubsection{Valves}

The pressure loss through a valve is calculated in different ways depending on the type of valve and the information that can be obtained from the manufacturer. If the pressure loss constant $\left(k_{v}\right)$ of a valve is known, then a more precise pressure loss calculation can be obtained using the following equation [2].

$$
\Delta p=\frac{\rho}{\rho_{w}\left(\frac{k_{v}}{\grave{V}}\right)^{2}}
$$

where $k_{v}$ is the valve constant. The density of water $\rho_{\text {water }}$ is found at $15^{\circ} \mathrm{C}$. $k_{v}$ is given for water and therefore the ratio between the hydrogen density and the water density is present. In eq. $28 \dot{V}$ is in $\mathrm{m}^{3} / \mathrm{h}$ and the pressure loss is in bar.

\subsubsection{Filter and mass flow meter}

Pressure losses in mass flow meters and filters in the system are calculated differently from pressure drops for valves. The pressure drop depends on a pressure loss constant $k_{p}$ and the cross-sectional area of the mass flow meter or filter; as the cross-sectional area is constant then the pressure loss constant $\left(k_{p}\right)$ can be obtained from the manufacturer. Equation 29 gives the pressure loss through a component with a constant crosssectional area and a given pressure loss constant [14].

$$
\Delta p=0.5 k_{p} \rho \dot{V}^{2}
$$

The volume flow rate $(\dot{V})$ is in $\mathrm{m}^{3} / \mathrm{h}$ and the pressure loss in bar.

\subsubsection{Tube and length equivalent pressure losses}

Pressure losses in the tubing and in components that are not covered by eqs. 28 and 29 can be found using the general pressure loss equation. The pressure loss depends on the friction factor and the length to diameter ratio. Pressure losses such as bends or ball valves are given in equivalent pipe length. The pressure loss for tubes is given by eq. 30

$$
\Delta p=\left(f \frac{L}{d}+k\right) \frac{\rho v^{2}}{2}
$$

where $k$ is the length equivalent of a component; for a straight tube $k=0$, but if a bend or a valve is present the approximate length equivalent value can be added. The length equivalent also enables the possibility to add many pressure losses together by just adding another pressure loss equivalent to eq. 30. Length equivalent values for different pressure losses can be found in textbooks. The friction factor $f$ is given by the Haaland equation, eq. 31 .

$$
\frac{1}{\sqrt{f}}=-1.8 \log \left(\left(\frac{\frac{\epsilon}{D}}{3.7}\right)^{1.11}+\frac{6.9}{\operatorname{Re}}\right)
$$

The friction factor is a function of Reynolds number (eq. 27 , the roughness $(r)$ of the tube and the diameter $(d)$. The roughness depends on the material of the tube and should be given by the manufacturer, or approximate values can be found in textbooks.

\subsection{Compression of hydrogen}

This section covers the compression of hydrogen. The equations used are real gas equations, and the efficiencies are found by general first estimate equations as efficiency curves for compressors are dependent on the type of compressor, the design of the compressor and the manufacturer.

\subsubsection{Compressor model}

The compressors considered are either reciprocating or centrifugal machines; for the compressors the heat loss is dependent on the type and manufacturer. A conservative approach assuming adiabatic compression has been used in the following models. There is a mass balance through the compressor $\left(\dot{m}_{1}+\dot{m}_{2}=0\right)$, the compression is adiabatic $(\dot{Q}=0)$, and there is work done on the hydrogen in the compressor corresponding to:

$$
W=\dot{m}\left(h_{\text {out }}-h_{\text {in }}\right)
$$


The pressure at the discharge side of the compressor is naturally higher than at the inlet so the momentum balance yields the pressure difference $\Delta p=p_{1}-p_{2}$, where $\Delta p$ is the pressure increase of the compression. In order to find the enthalpy out of the compressor, the mass flow and the discharge pressure need to be known. The mass flow of the compressor is calculated by defining the volume of the cylinders $\left(V_{c y l}\right)$, piston strokes per second $(n)$ and a defined function for the volumetric efficiency $\eta_{v}$.

$$
\dot{m}=V_{c y l} \cdot \rho_{\text {in }} \cdot \eta_{v} \cdot n
$$

For this model the volumetric efficiency has the highest efficiency of $90 \%$ at a pressure ratio of unity, and it is decreasing with $5 \%$ per increased pressure ratio across the compressor. The energy balance of the compressor is the enthalpy flow into the compressor, the enthalpy flow out of the compressor and the power added in the compressors shown in eq. 32 . The enthalpy of the discharge of the compressor is found from eq. 34 since the compression is assumed to be adiabatic.

$$
h_{\mathrm{out}}=\frac{h_{o u t, \eta}-h_{\mathrm{in}}}{\eta}-h_{\mathrm{in}}
$$

where $\eta$ is the isentropic efficiency and $h_{\text {out }, \eta}$ is the enthalpy for an isentropic compression. A general estimate of the isentropic efficiency is given by eq. 35] [32].

$$
\begin{aligned}
\eta_{\text {is }}=0.1091 \cdot \log & \left(\frac{p_{\text {out }}}{p_{\text {in }}}\right)^{3}-0.5247 \cdot \log \left(\frac{p_{\text {out }}}{p_{\text {in }}}\right)^{2} \\
& +0.8577 \cdot \log \left(\frac{p_{\text {out }}}{p_{\text {in }}}\right)+0.3727
\end{aligned}
$$

where $p_{\text {in }}$ is the suction pressure and $p_{\text {out }}$ is the discharge pressure. Equation 35 is valid in the range $1.1<\frac{P_{\text {out }}}{p_{\text {in }}}<5$. The pressure ratio never exceeds 5 in the calculations.

\subsection{Additional models}

The following section considers different types of components that have not yet been described. The components include heat exchangers and flow mixers.

\subsubsection{Heat balance model}

The model of the heat exchanger is done as a heat balance equation transferring heat from one fluid stream to another. The cooling capacity is calculated by eq. 36

$$
Q=\dot{m}\left(h_{\text {in }}-h_{\text {out }}\right)
$$

where $Q$ is the cooling demand.

\subsubsection{Mixer models}

The mixers (or splitters) are components with three or more inlets/outlets allowing several streams to merge into one stream or split one stream into several streams.

There are two different kinds of mixers: ideal mixing in a point and ideal mixing in a volume. Ideal mixing in a point mixes the streams in a black box, only considering the mass flow in and out, the enthalpy in and out and the pressure. The ideal mixing in a volume considers the mixing inside a small volume which corresponds to a tank with three entrances or exits.

\section{Mixing without a volume}

The mass balance of the streams entering the mixer is $m_{1}+m_{2}+m_{3}=0$. The pressure of the streams must be the same $p_{1}=p_{2}=p_{3}$. The energy balance for the mixer is as from eq. 37

$$
\frac{d E}{d t}=\dot{m}_{1} h_{1}+\dot{m}_{2} h_{2}+\dot{m}_{3} h_{3}
$$

As the mixing is ideal and there is mass balance the change in energy in the mixer is $\frac{d E}{d t}=0$.

\section{Mixing with a volume}

The mixer with a volume corresponds to a tank with three entrances. The difference between the described tank model and the mixer with a volume is found in the mass balance eq. 8 and the energy balance eq. 11 . The mass and the energy balance equation for three entrances are given by eq. 38 and eq. 39. respectively.

$$
\begin{gathered}
V \frac{d \rho}{d t}=\dot{m}_{1}+\dot{m}_{2}+\dot{m}_{3} \\
\frac{d h}{d t}=\frac{1}{M} \cdot\left(h_{1} \frac{d m_{1}}{d t}+h_{2} \frac{d m_{2}}{d t}+h_{3} \frac{d m_{3}}{d t}+V \frac{d p}{d t}\right)
\end{gathered}
$$

The heat loss from the mixer has been considered negligible from a system perspective. The pressures at the port and in the mixer are all the same, $p=p_{1}=p_{2}=p_{3}$.

\subsection{Energy optimization}

The optimization of hydrogen fueling stations with respect to energy usage can be done in different ways. Studies of different parameters, such as the number of tanks in the fueling stations cascade system, the pressure in the tanks and the volume of the tanks, can outline the best setup of the station. The energy savings obtained in the compressor and heat exchangers by adding another tank or changing the pressure or volume can be directly found, and the trade-off between investment cost and running costs can be used to find the best setup for the manufacturer of the station without compromising security of supply. 


\section{Verification and validation of the model}

This section compares the developed empirical model to a model developed based on confidential, experimental data derived from an actual hydrogen fueling station. The results are compared with respect to temperatures, pressures and mass flow rates at different places in a fueling station.

\subsection{Comparison of models}

The two models compared are the generic model (GM) developed with the equations presented in this work and a specific model (SM) developed by the company NEL hydrogen using manufacturer data. Both models simulate the fueling of a vehicles according to the fueling protocol, the SAE J2601. The results shown here are for an A70 fueling of a $0.161 \mathrm{~m}^{3}$ tank with an initial pressure of $20 \mathrm{bar}$. The three tanks in the cascade system at the station are all pressurised to 950 bar. The tanks are of the same materials and dimensions in both models. The average pressure ramp rate for both systems was set at the exit of the fueling station. Figure 1

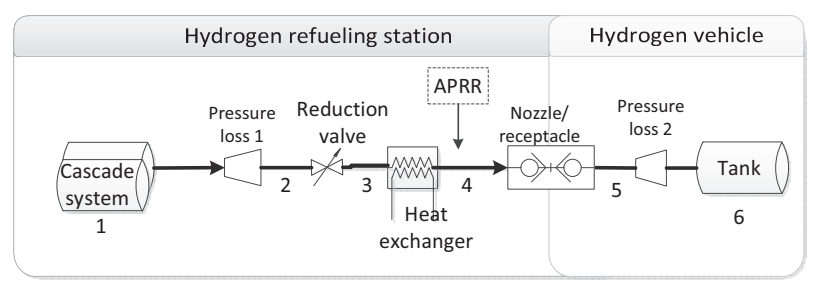

Figure 1: Sketch of a fueling station indicating state points shown in Figure 2 Figure 3 Figure 5 and Figure 6

shows a simplified sketch of a fueling system. Pressure losses have been collected in two components for simplicity. The state points used in Figure 11 corresponds to those in Figure 2 and 3 as legends. Point (1) is in the tanks in the cascade system, point (2) is before the reduction valve, point (3) is after the reduction valve, point (4) is after the heat exchanger and just before the outlet of the station, point (5) is after the receptacle in the vehicle and point (6) is inside the vehicle tank. In the figures results from the specific model are denoted by legend (SM) and the data from the generic model are labelled by legend (GM). Figure 2 shows that the pressure development during a fueling process is almost identical for the two models. The largest pressure deviation is 7 bar (point 2), which is acceptable when considering the large pressure losses that are present in the system, see figure 2(b)

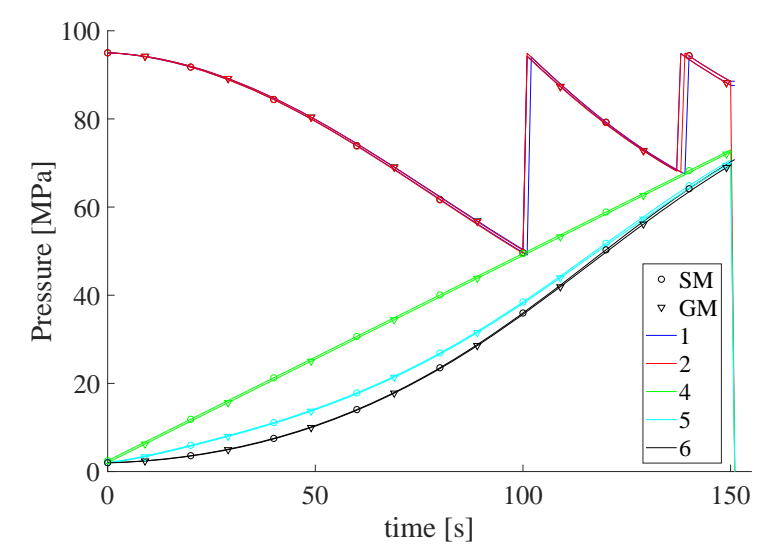

(a) Pressures in the system during a fueling.

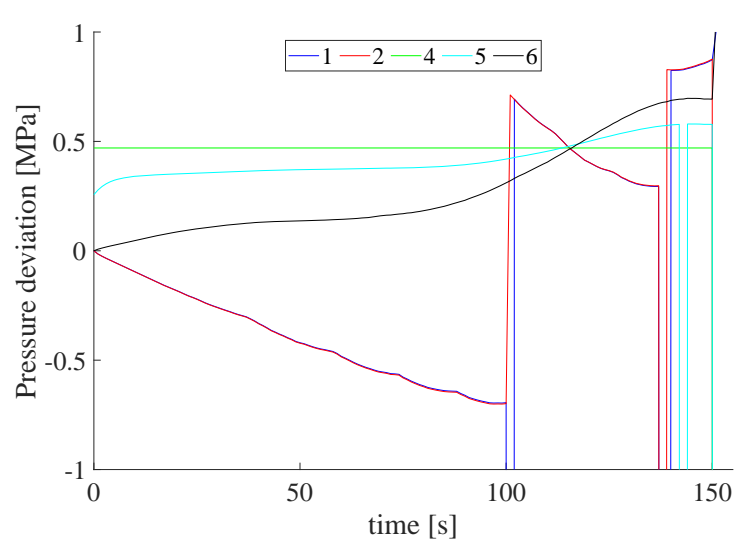

(b) Deviation in pressure between the two models.

Figure 2: Development of pressures in the fueling station during a fueling. (SM) specific model, (GM) generic model. The numbers refer to the placement of the data with respect to fig. 1]

The temperature development during fueling for both models can be seen in Figure 3. The temperature development are similar for both models and the largest difference is in the vehicle tank with approximately $3^{\circ} \mathrm{C}$ difference at its peak. Comparing Figure 2(a) of the pressure and Figure 3(a) of the temperature, it is seen that a pressure drop leads to a temperature rise of the hydrogen, which is due to the Joule-Thomson effect. The mass flow of the two simulations is shown in Figure 4(a) The specific model has a slightly higher mass flow rate for the first 120 seconds before the two curves cross and the generic model has a higher mass flow rate. Considering the deviation it is approximately 1 $\mathrm{g} / \mathrm{s}$, which is less than $2 \%$ of the peak mass flow rate at just over $50 \mathrm{~g} / \mathrm{s}$.

The two models, which are developed independently of each other, give very similar results. The deviations 


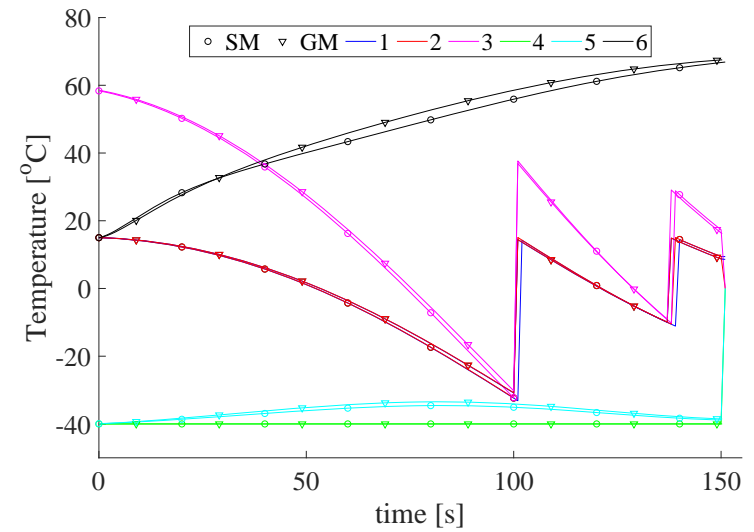

(a) Temperatures in the system during a fueling.

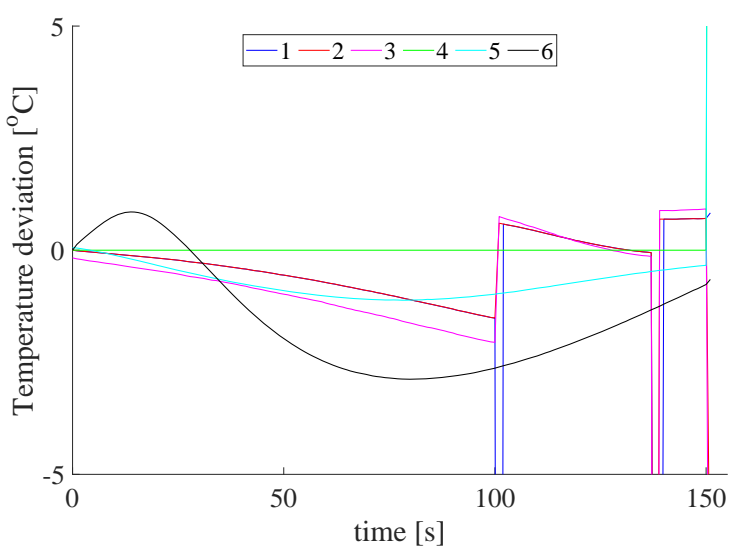

(b) Deviation in temperature between the two models.

Figure 3: Development of temperatures in the fueling station during a fueling. (SM) specific model, (GM) generic model. The numbers refer to the placement of the data with respect to fig. 1

between them are small, and both models perform as expected. The specific model has been successfully validated as NEL hydrogen has performed a number of tests which have been compared to their model with success.

\subsection{Comparison to test data}

The following section considers data from a hydrogen fueling of a 700 bar tank. The data was extracted in 2011 from a NEL hydrogen fueling station. Pressures and temperatures were measured at different places in the system. The temperature data obtained are limited to before and after a heat exchanger placed after the reduction valve in the system. The pressure was measured at the outlet of each tank and before and after the reduction valve. In addition, a mass flow meter measured the mass flow rate in the system. The data from the test is compared to a simulation using the generic model which fol-

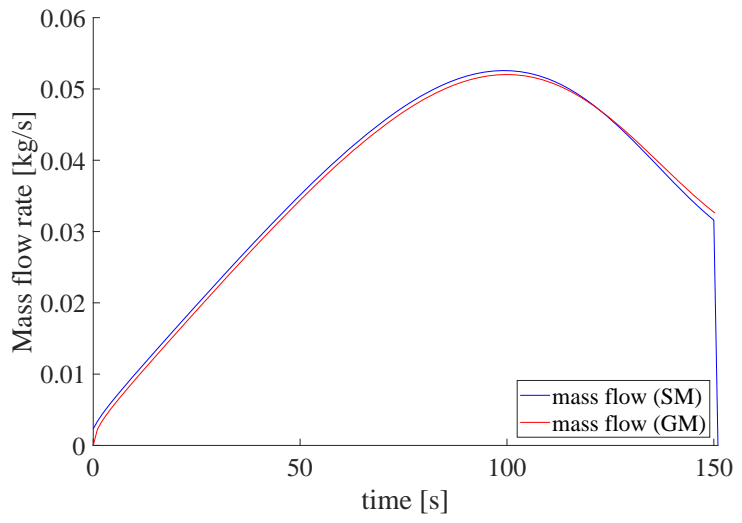

(a) The mass flow rate during a fueling.

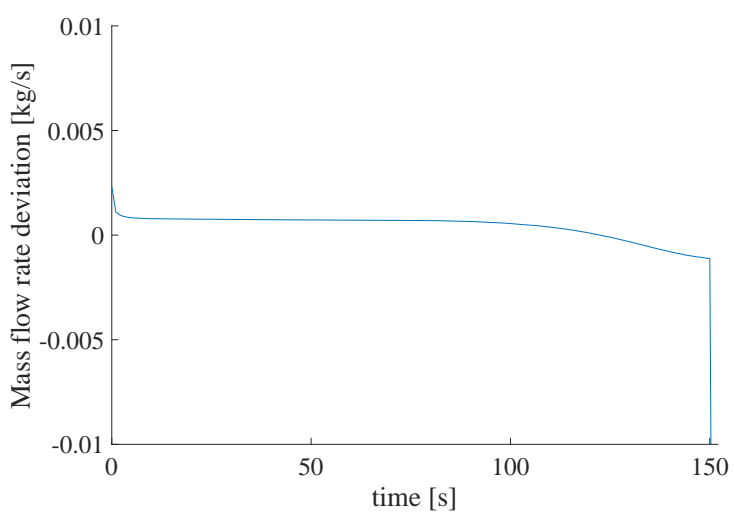

(b) Deviation in mass flow rate between the two models.

Figure 4: Development of mass flow rates in the fueling station during a fueling. (SM) specific model, (GM) generic model.

lows the lookup table based fueling protocol. Hence, the average pressure ramp is constant and the temperature out of the heat exchanger is $-40{ }^{\circ} \mathrm{C}$ (point 4 in Figure 1 ). The model used for the simulations matches the real station. Not all data were given for the tested fueling station so, assumptions of pressure losses had to be made for the simulation. The simulation model was fitted as close to the measured data as possible, while completing an A70 fueling according to the protocol. The tanks at the station are the same size as the tank in the vehicle. The pressure losses in the simulation were fitted to the data by changing the pressure loss constants. The tested fueling station had two heat exchangers, whereas only information on the second one placed after the reduction valve was available. The model also had two heat exchangers which both were cooling the hydrogen to $-40{ }^{\circ} \mathrm{C}$. The average pressure ramp rate used in the generic model was changed, so the average ramp rate for both the model and the test were the same, when a 
complete fueling is considered. Figure 5(a) shows the

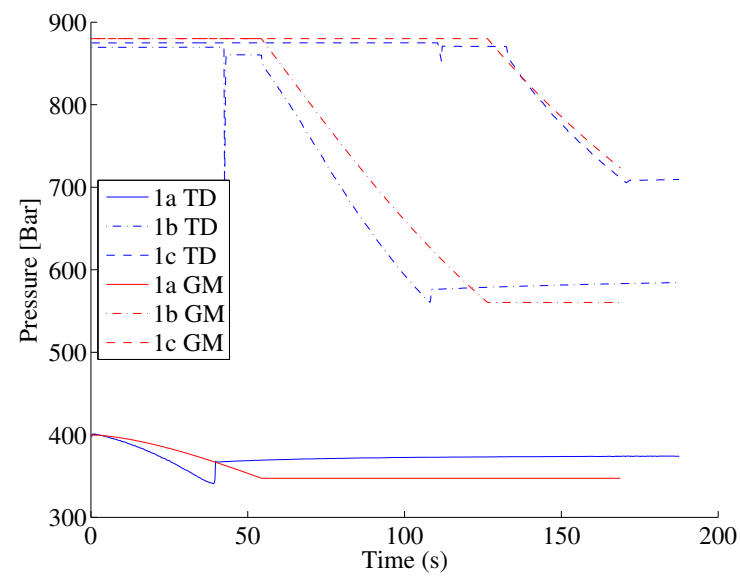

(a) Pressure out of the tanks

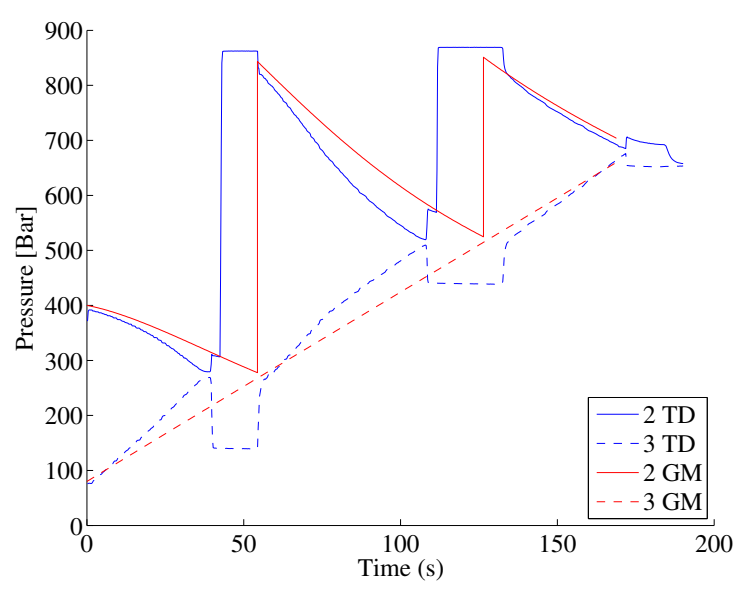

(b) Pressure on each side of the reduction valve

Figure 5: Pressure comparison of the generic simulation model and the data from a hydrogen fueling

measured pressure out of the tanks at the fueling station compared to a simulated pressure of a fueling. The tanks have the same initial pressure for both the test and the model. The final pressure in each tank is also approximately the same. The change between tanks at the hydrogen fueling station is different as it stops the fueling for some seconds while changing tank, this results in an average pressure ramp that is not constant for the test as it is for the simulation. However, the final pressure in each tank is the same, and hence the same amount of mass is removed from the tanks for both the test and the simulation. Figure 5(b) shows the pressure on both sides of the reduction valve. It is seen that the average pres- sure ramp rate is the same for both the test and the simulation for a complete fueling. The fueling stops during the test at each tank shift, which results in a break and some waiting time which is not present in the simulation. The shift between the tanks at the station happens at approximately the same pressure in the test and in the simulation. The temperature, shown in Figure 6 , into

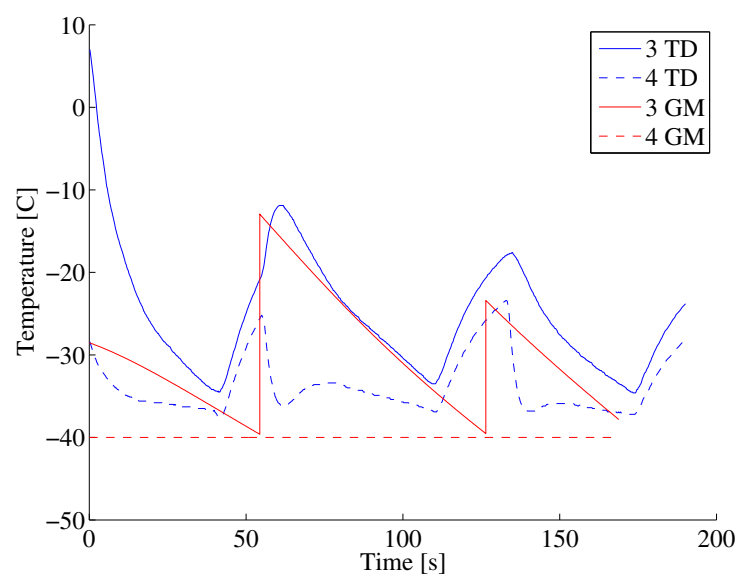

Figure 6: The temperature into and out of the heat exchanger for both the test data and the simulation of a fueling

and out of the heat exchanger follows the same pattern. In the simulation the hydrogen is cooled down to -40 ${ }^{\circ} \mathrm{C}$ before the valve, and for the test the inlet temperature to the reduction valve is unknown. The comparison shows that the pattern of the temperature before and after the heat exchanger is similar for the test and the model. The differences should be found in the cooling capacity of the hydrogen in the test, which is less than needed by the demand for cooling to $-40{ }^{\circ} \mathrm{C}$. As the hydrogen is cooled down before the reduction valve, the inlet temperature to the heat exchanger showed in Figure 6 is a consequence of the Joule-Thomson effect created by the pressure losses across the valve. Again, it should be noted that the actual test was not carried out for the purpose of experimental data and therefore there are various other unknown factors that have not been considered in the simulations. The mass flow of the test and the simulation can be seen in Figure 7. The mass flow rate seems a little higher for the simulated fueling; this is likely due to an inaccuracy in a tank dimension or the pressure loss in the vehicle is set too low. The two mass flow rates show similar trends, despite the test results have the break in fueling when switching tanks in the cascade system.

The comparison between the test data and the sim- 


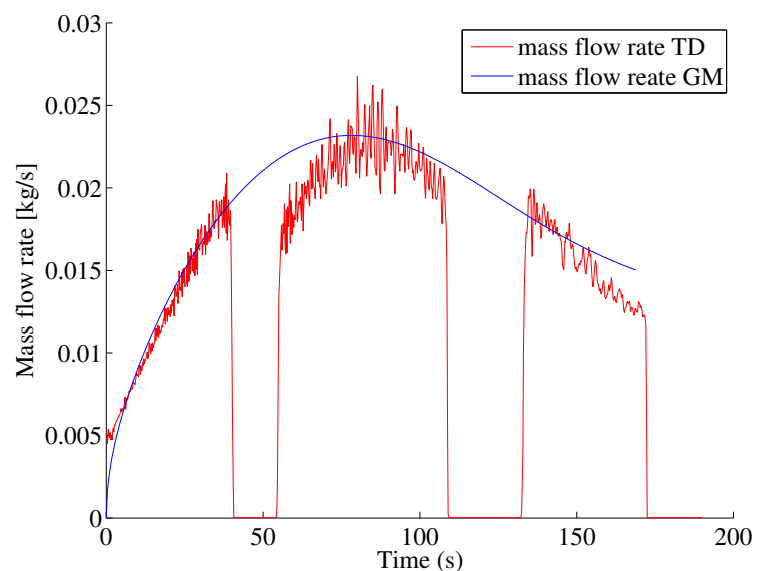

Figure 7: The mass flow rate for both the test data and the simulation of a fueling.

ulation shows that the trends of the generic model are similar to a real hydrogen fueling. The pressure drops, the Joule-Thomson effect and the temperatures seem to behave similarly in the simulation and in the test. Furthermore, the pressure in the tanks at the station end at the same pressure as for the test data as does the outlet of the reduction valve. The comparison shows that the generic model for hydrogen fueling can simulate hydrogen fueling scenarios close to real-life hydrogen fueling.

\section{Temperature development in the compressed hy- drogen storage system as function of pressure loss and fueling time}

Using the generic thermodynamic simulation model it is possible to investigate complete hydrogen fuelings. A general concern and safety issue when fueling hydrogen vehicles is the temperature of the hydrogen inside the compressed hydrogen storage system (CHSS) as the storage tank has a temperature limit of $85^{\circ} \mathrm{C}$ according to SAE J2601. The temperature increase inside the CHSS is primarily due to heat of compression and the temperature of the hydrogen entering the CHSS. The main driver of the temperature increase is the magnitude of the enthalpy entering the CHSS, which can be directly correlated to the pressure and temperature at the outlet of the station. The pressure at the station outlet is corresponding to the pressure in the vehicle tank added to the pressure loss between the station outlet and the storage tank in the CHSS, thus the pressure losses in the vehicle. The temperature at the outlet of the station is depending on the temperature the refrigeration facility cools the hydrogen at; typically designed for $-40{ }^{\circ} \mathrm{C}$. The pressure losses present in the CHSS were all assumed to be isenthalpic processes. Thus the pressure and temperature changes between the outlet of the station and the CHSS storage tank, although the enthalpy of the stream remains constant. This study shows how the pressure losses between the station outlet and the vehicle storage tank indirectly influences the fueling process.

When filling a hydrogen vehicle there are restrictions to the mass flow rate, maximum pressure and temperature in the CHSS. The fueling proceeds depending on several parameters such as starting pressure and temperature in the CHSS, the type of storage vessel, the cooling the hydrogen refueling station (HRFS) can deliver, ambient temperature and the presence of communication between the CHSS and the HRFS. These data are used to make sure that the safety in the CHSS is not compromised during a fueling. Designing and producing a HRFS can be done with full control of all processes inside the HRFS including the average pressure ramp rate (APRR) and the cooling of the hydrogen. The only variable the fueling station can not be designed specifically for, is the magnitude of the pressure loss inside the CHSS. The HRFS can be state of the art both by means of fueling time, dimensioned final state of charge and energy consumption but the influence of the CHSS can only be assumed and the pressure losses in some CHSS might not be a good match for the designed station and the station might not operate as designed.

The studied hydrogen fueling system is a simplification of a station as shown in figure 1 . The study assume 3 different pressure losses in the vehicle and 5 different APRRs (5 MPa/min, $20 \mathrm{MPa} / \mathrm{min}, 35 \mathrm{MPa} / \mathrm{min}, 50$ $\mathrm{MPa} / \mathrm{min}, 65 \mathrm{MPa} / \mathrm{min}$ ) fueling a vehicle from $5 \mathrm{MPa}$ to $80 \mathrm{MPa}$ each time. The pressure losses chosen is a high pressure loss (HPL) corresponding to a maximum pressure loss of $30 \mathrm{MPa}$ in the CHSS during a fueling of a $0.172 \mathrm{~m}^{3}$ vessel at an APRR of $28.5 \mathrm{MPa} / \mathrm{min}$. A medium pressure loss (MPL) which at the same conditions corresponds to a pressure loss of $15 \mathrm{MPa}$. The last case is a CHSS with no pressure loss (NPL) under the same conditions. As shown in section 2.5 the pressure losses are a function of the volume flow and density, thus the highest pressure loss occurs at the highest mass flow rate. Lowering the APRR but keeping the rest of the system constant will result in lower pressure losses as the mass flow rate is reduced with the increasing fueling time. The final pressure for all the different cases is shown in figure 8, it can be seen that all the simulated fuelings end at a pressure of $80 \mathrm{MPa}$ in the CHSS 
and that the different APRRs results in different fueling times. The longest fueling time was found for the lowest APRR and vice versa. Keeping the APRR constant, it also shows that the higher the pressure loss in the vehicle the longer the fueling time. This is because the APRR is regulated at the HRFS outlet and with the higher pressure loss in the CHSS forces the pressure at the outlet of the station to be higher than for a CHSS with no pressure losses, fuelling to the same pressure. It therefore takes longer time to reach the final pressure at $80 \mathrm{MPa}$ in the CHSS as the fueling needs to proceed to a higher pressure at the station outlet due to the pressure losses in the CHSS. Figure 9 shows the final tempera-

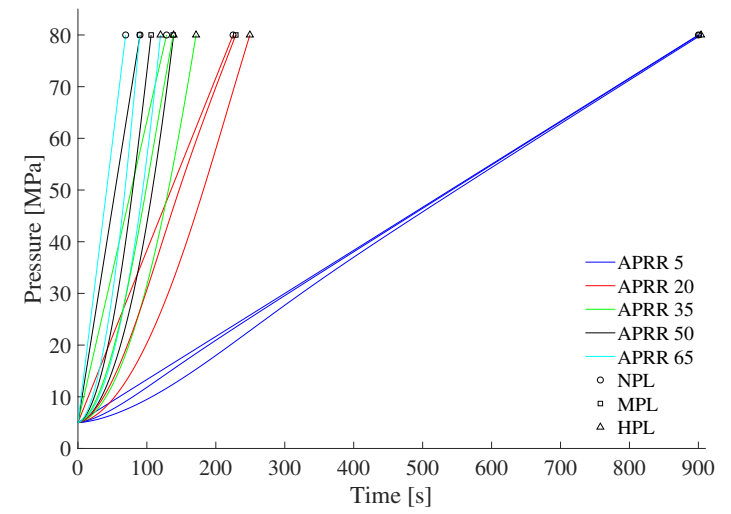

Figure 8: Final pressure and fueling time for the three pressure loss cases and 5 APRRs; no pressure loss (NPL), medium pressure loss (MPL) and high pressure loss (HPL).

ture as a function of the APRR and the highest pressure loss during the fueling as a function of the APRR. Figure 9(a) shows two important properties during fueling. First it is seen that if there is no pressure loss present in the vehicle, the temperature in the CHSS is the same no matter the APRR, as long the hydrogen leaves the station at $-40{ }^{\circ} \mathrm{C}$. The second important property is that the temperature in the CHSS increases with increasing pressure loss in the vehicle, figure 9(b), and when a pressure loss is present in the vehicle, the temperature in the CHSS also increases as the APRR increases. In order to fully understand why the pressure loss in the vehicle influences the temperature in the CHSS it is necessary to take a closer look at the enthalpy at the exit of the station and entrance at the CHSS. Figure 10(a) shows the enthalpy at the outlet of the station for each pressure loss and APRR, the circle, square and triangle denotes the enthalpy at the station outlet when the fueling finishes for no pressure loss fueling, medium pressure loss fueling and high pressure loss fueling, respectively. The

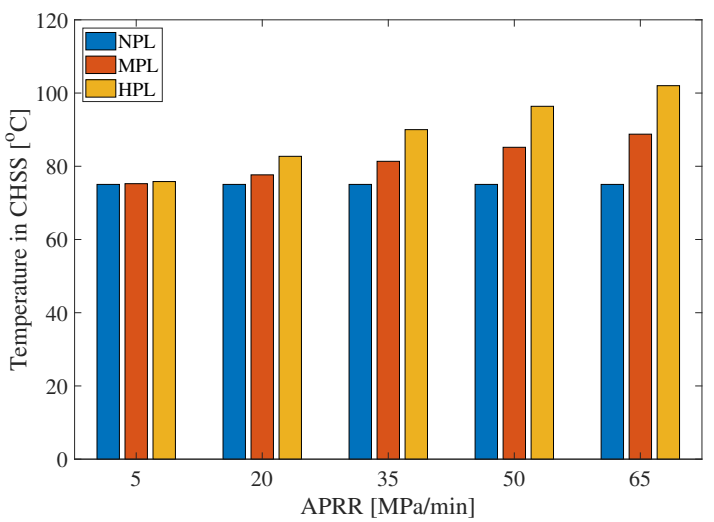

(a) Final temperature in CHSS

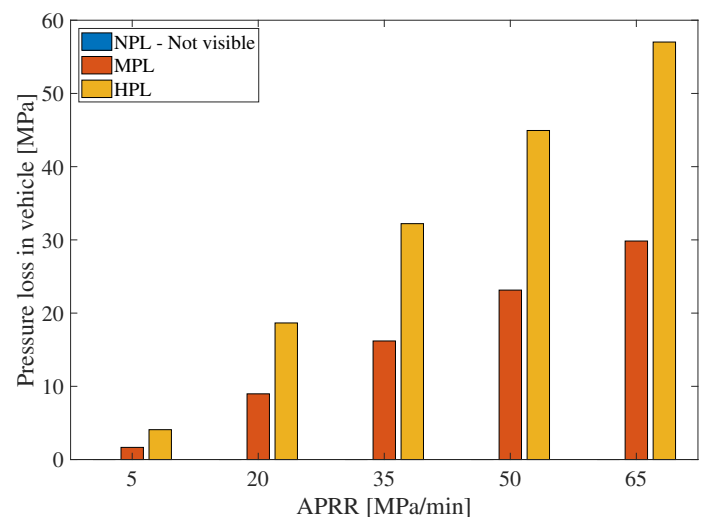

(b) Pressure loss between receptacle and hydrogen vessel in vehicle

Figure 9: Temperature and pressure loss as a function of APRR and pressure loss coefficient in vehicle; no pressure loss (NPL), medium pressure loss (MPL) and high pressure loss (HPL). NPL is not visible as it is zero for all APRRs

reason why the final enthalpy is not the same in all cases even though the temperature at the outlet of the station is constant at $-40^{\circ} \mathrm{C}$, is that the pressure at the station outlet in general needs to be higher as the pressure loss in the vehicle increases. The enthalpy out of the station depends on the APRR and the pressure loss in the vehicle. The difference in final enthalpy is therefore a result of the higher pressure at the station outlet which is needed to overcome the pressure loss in the vehicle shown in fig. 9(b) As the main driver of the temperature increase of the CHSS is heat of compression, the higher enthalpy directly result in the higher temperature.

A last important parameter to show when considering the pressure loss in the vehicle is the final mass inside the CHSS as shown in fig. 10(b) The mass depends on the density and the volume of the CHSS, the 


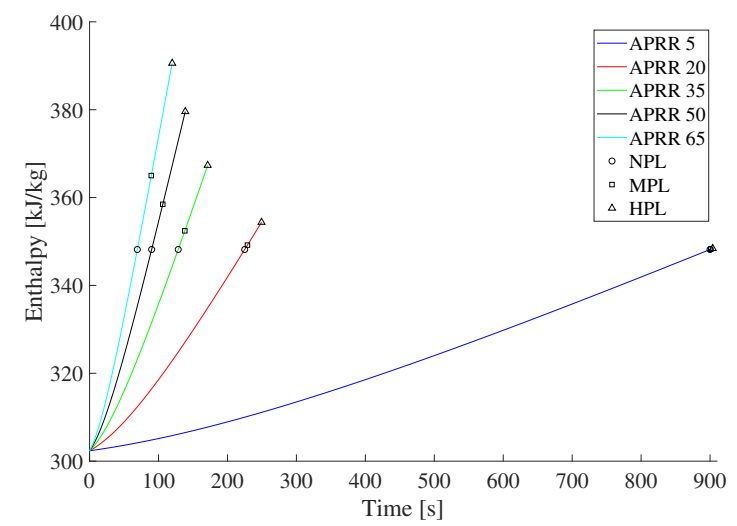

(a) Enthalpy at station outlet during a fueling. Circle, square and triangles shows the enthalpy when the fueling finishes for NPL, MPL and MPL, respectively.

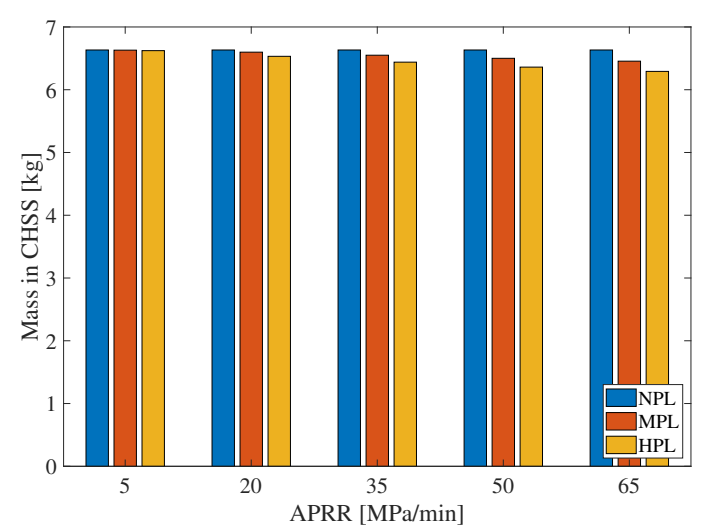

(b) Mass in CHSS at end of filling

Figure 10: Enthalpy and mass as a function of APRR and pressure losses in vehicle; no pressure loss (NPL), medium pressure loss (MPL) and high pressure loss (HPL).

volume is the same for all the cases. The density however is a function of enthalpy, temperature and pressure, so as the temperature and enthalpy inside the CHSS is higher and the final pressure is constant, the density will be lower with increasing temperatures and thus will the filled mass at the end of the fueling be lower.. This is shown in fig. 10(b) were higher pressure losses results in higher temperatures in the CHSS (fig. 9(a) which results in a lower amount of fuelled hydrogen. Figure 11 shows the final temperature inside the CHSS at different outlet temperatures of the HRFS and with no pressure losses in the vehicle. This causes the minimum temperature increase of an adiabatic tank and the figure shows the importance of the cooling at the hydrogen fueling station. The final temperature will be higher as shown

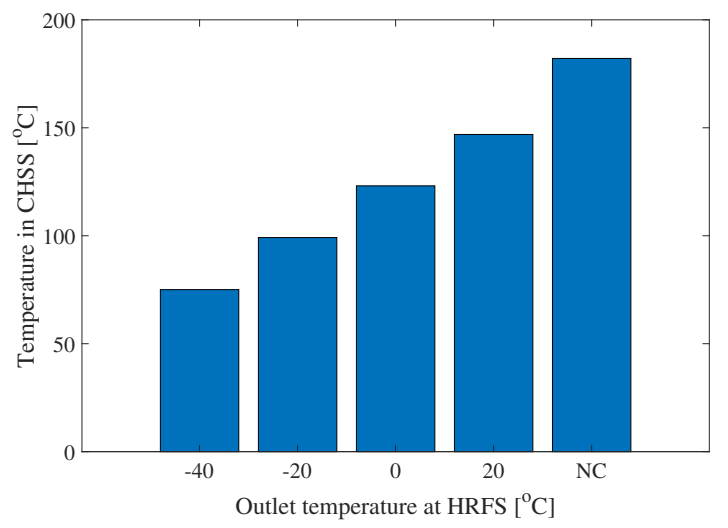

Figure 11: Final temperature in CHSS at different pre-cooling outlet temperatures and no cooling (NC) for the case were no pressure loss is present in vehicle.

in fig. 9(a) when pressure losses are present in the vehicle.

\section{Discussion}

The generic model for hydrogen fueling has been verified and validated. The result of the comparison between the specific model and the generic model for the verification was expected, as both models are using the same thermodynamic principles for the different component. The more specific equations for pressure losses, heat transfer, fueling protocol could have caused differences in the models as they are not necessarily the same and integrated into the simulation in different ways, especially the heat transfer which in the generic model is calculated as transient heat transfer through a wall, is in the specific model simpler. The validation shows the same trends between the test data for a fueling and the generic model. The system used for obtaining the test data is not known in detail, but the main parameters such as tank sizes, and target temperature of the cooling facility was given. The generic model was therefore fitted to match the test data with respect to the size of the pressure losses and the average pressure ramp rate. The values used in the generic model in order to match the trends of the test case all seems reasonable and within the boundaries of what could be expected.

The thermodynamic analysis of the pressure losses effect on the development of the temperature in the CHSS shows that the pressure loss in the vehicle between the exit of the station and the storage tank influences the temperature increase of the storage tank. The primary 
driver of the temperature increase is heat of compressions which is a function of the pressure and enthalpy. As the pressure at the station outlet increases with increasing pressure losses in the vehicle the temperature inside the storage tank increases even though the outlet temperature of the station is constant at $-40{ }^{\circ} \mathrm{C}$. The pressure increases at the station outlet is leading to increasing enthalpies and therefore to a higher temperature due to heat of compression inside the storage tank. The main safety concern is the temperature inside the storage tank in the vehicle exceeding $85{ }^{\circ} \mathrm{C}$. The study shows that the lower the pressure loss in the vehicle, the lower the final temperature inside the vehicle no matter the APRR. If it is possible for the pressure loss in the vehicle to be known by the station, the APRR could be adjusted accordingly allowing faster fueling. By fueling faster the pressure loss will increase and the pressure at the outlet of the station would need to be higher. This could result in a higher pressure needed in the storage system at the station, which would require more energy to refill. Although, this would depend on the existing system design where the pressure today already is higher than needed at the cascade system in the station.

\section{Conclusion}

The purpose of this study can be divided into two: first this study explains, verifies and validates the method used in the generic simulation model implemented in the freely available hydrogen library for Dymola. Second the generic model is used to show how the pressure losses in the vehicle influences the fueling process, the final temperature in the CHSS and the final outlet pressure at the station as well as the total mass filled.

The comparison of the specific model with the generic model showed almost identical results. The differences were negligibly small taking into account that the models use different equations and different solvers. The comparison between the data of a real hydrogen fueling station and the model showed that the model behaves quite close to a real fueling station even though the model is uses some simplifications. The largest difference between the data and the model used, was that during a real fueling there is a break when changing the tank at the station.

The temperature into the heat exchanger followed the same pattern for the model as for the test data, though the temperature in the vehicle tank was different as the test did not cool down to $-40{ }^{\circ} \mathrm{C}$. The mass flow rates were similar too, as for the model and the data. The comparison between the specific model, the generic model and the test data from a real fueling shows that the generic model works as expected. The presented methodology can therefore be used for dynamic simulations of hydrogen fueling stations.

The analysis of the temperature development inside the CHSS as a function of pressure loss and fueling time was done using the generic model. The study showed that if there are no pressure losses in the vehicle the final temperature in the CHSS is constant no matter the APRR. If there are pressure losses in the CHSS the lower the APRR the lower the temperature increase, as the pressure loss is directly related to the mass flow rate which is decreased by lower APRRs. The study shows the importance of limiting the pressure loss in the vehicle in order to obtain faster fueling times increasing the APRR without compromising the boundary conditions. The study also shows the importance of cooling hydrogen at the station outlet, showing that the minimum temperature increase in the CHSS is arounf $75^{\circ} \mathrm{C}$ with cooling to $-40{ }^{\circ} \mathrm{C}$ and that the minimum temperature in the CHSS increases to $180{ }^{\circ} \mathrm{C}$ if there is no cooling present.

\section{Acknowledgements}

The authors would like to thank the Danish Energy Agency for financial support for project Link2009 project number: 64009-0172 and our industrial partner, NEL Hydrogen, for collaboration.

\section{Nomenclature}




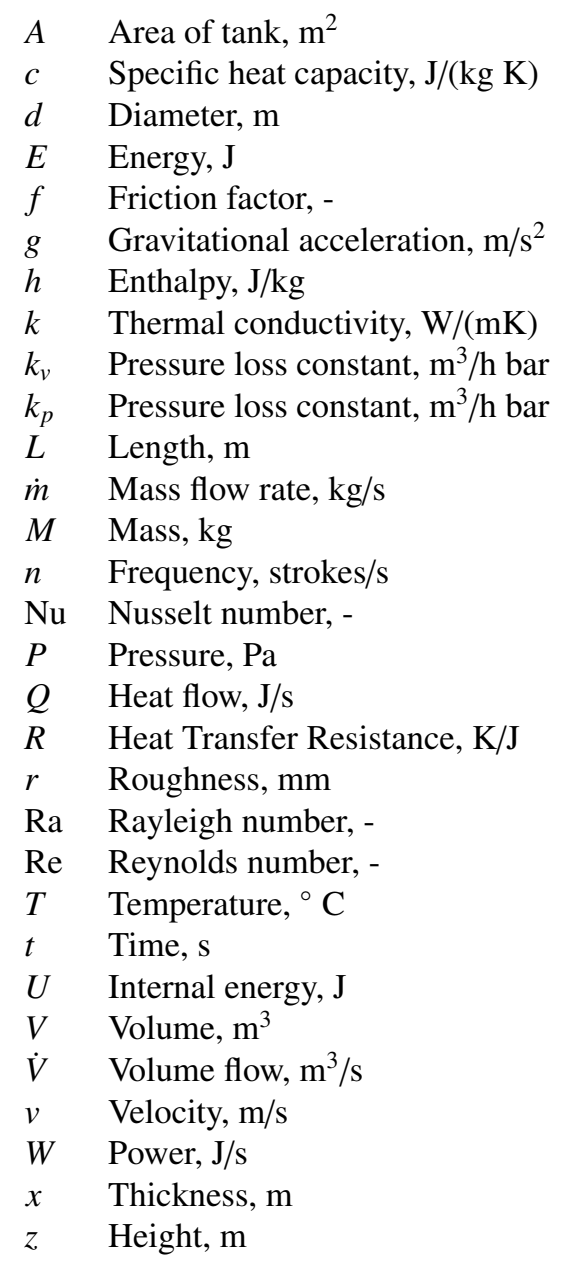

\section{References}

[1] Abhilash S, Kim H, Setoguchi T. Three dimensional numerical computations on the fast filling of a hydrogen tank under different conditions. Int J Hydrogen Energy 2012;37:7600-7611.

[2] Burkert. Data Sheet. 2013. http :

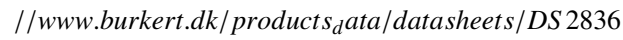
Standard -EU - EN.pdf. 1, The 10th of June 2013.

[3] Daney DE. Turbulent natural convection of liquid deuterium hydrogen and nitrogen within enclosed vessels. Int J Heat Mass Tran 1976;19:431-441.

[4] Bourgeois T, Brachmann T, Barth F, Ammouri F, Baraldi D, Melideo D, Acosta-Iborra B, Zaepffel D, Saury D, Lemonnier D. Optimization of hydrogen vehicle refuelling requirements. Int J Hydrogen Energy 2017;42:13789-13809.

[5] Dicken CBJ, Mérida W. Measured effects of filling time and initial mass on the temperature distribution within a hydrogen cylinder during refueling. J Power Sources 2006;165:324-336.

[6] Dixon S. Fluid dynamics and thermodynamics of turbomachinery. 4 ed. Butterworth-Heinemann; 1998, p. 15-17.

[7] Elgowainy A, Reddi K, Lee D, Rustagi N, Gupta E. Technoeconomic and thermodynamic analysis of pre-cooling systems at gaseous hydrogen refueling stations. Int J Hydrogen Energy 2017;42:29067-29079.

[8] Farzaneh-Gord M, Deymi-Dashtebayaz M, Rahbari H, Niaz-

$\begin{array}{ll}\text { Greek } & \\ \alpha & \text { Heat transfer coefficient, } \mathrm{W} /\left(\mathrm{m}^{2} \mathrm{~K}\right) \\ \beta & \text { Efficiency },- \\ \eta & \text { Heat capacity ratio, - } \\ \gamma & \text { Dynamic viscosity, } \mathrm{kg} /(\mathrm{sm}) \\ \mu & \text { Density, } \mathrm{kg} / \mathrm{m}^{3} \\ \rho & \\ & \\ \text { Subscript } & \text { Ambient } \\ a & \text { Cylinder } \\ \text { cyl } & \text { Gas } \\ g & \text { Node number } \\ i & \text { Into component } \\ \text { in } & \text { Isentropic } \\ \text { is } & \text { Conductivity } \\ k & \text { Total thickness } \\ L & \text { Out of component } \\ \text { out } & \text { Pressure } \\ P & \text { Volumetric } \\ v & \text { Wall } \\ w & \text { Water properties at } 15^{\circ} \mathrm{C} \\ \text { water } & \end{array}$

Abbreviations

APRR Average pressure ramp rate

CHSS Compressed hydrogen storage system

HRFS Hydrogen refueling station

GM Generic Model

HPL High pressure loss

MPL Medium pressure loss

NPL No pressure loss

SAE Society of Automotive Engineers

SM Specific Model

SOC State of charge

TD Test data

TIR Technical information report

mand $\mathrm{H}$. Effects of geometry and inconsistent mass flow rate on temperature within a pressurized cylinder during refueling. Int J Hydrogen Energy 2012;37:6043-6052.

[9] Galassi C, Baraldi D, Iborra BA, Moretto P. CFD analysis of fast filling scenarios for 70 mpa hydrogen type 4 tanks. Int $\mathrm{J}$ Hydrogen Energy 2012;37:6886-6892.

[10] Heitsch M, Baraldi D, Moretto P. Numerical investigations on the fast filling hydrogen tanks. Int $\mathrm{J}$ Hydrogen Energy 2010;36:2606-2612.

[11] Hosseini M, Dincer I, Naterer G, Rosen MA. Thermodynamic analysis of filling compressed gaseous hydrogen storage tanks. Int J Hydrogen Energy 2012;37:5063-5071.

[12] Incropera F, DeWitt D, Theodore B,Lavine A. Introduction to heat transfer. 5 ed. New York John Wiley \& Sons; 2007.

[13] Kaviany M. Principles of Heat Transfer. 1 ed. New York John Wiley \& Sons; 2002, p. 294-296. 
[14] Krex H. Maskin staabi. 9 ed. Copenhagen Nyt Teknisk Forlag; 2004, p. 143-151.

[15] Li Q, Zhou J, Chang Q, Xing W. Effects of geometry and inconsistent mass flow rate on temperature within a pressurized cylinder during refueling. Int J Hydrogen Energy 2012;37:60436052 .

[16] Maus S, Hapke J, Ranong C, Wuchner E, Friedlmeier G, Wenger D. Filling procedure for vehicles with compressed hydrogen tanks. Int J Hydrogen Energy 2008;33:4612-4621.

[17] Miguel N, Acosta B, Baraldi D, Melideo R, Ortiz Cebolla $\mathrm{R}$, Moretto P. The role of initial tank temperature on refuelling of on-board hydrogen tanks. Int J Hydrogen Energy 2016;41:8606-8615.

[18] Monde M, Mitsutake Y, Woodfield P, Maruyama S. Characteristics of heat transfer and temperature rise of hydrogen during rapid hydrogen filling at high pressure. Heat Transfer - Asian Research 2007;36:13-27.

[19] Monde M, Woodfield P, Takano T, Kosaka M. Estimation of temperature change in practical hydrogen pressure tanks being filled at high pressure of 35 and $70 \mathrm{MPa}$. Int J Hydrogen Energy 2012;37:5723-5734.

[20] Ozsaban M, Midilli A, Setoguchi T. Exergy analysis of a high pressure multistage hydrogen gas storage system. Int J Hydrogen Energy 2011;36:11440-11450.

[21] Reddi K, Elgowainy A, Sutherland E. Hydrogen refueling station compression and storage optimization with tube-trailer deliveries. Int J Hydrogen Energy 2014;39:19169-19181.

[22] Reddi K, Elgowainy A, Rustagi N,Gupta E. Impact of hydrogen SAE J2601 fueling methods on fueling time of light-duty fuel cell electric vehicles. Int J Hydrogen Energy 2017;39:1667516685 .

[23] Rothuizen E, Merida W, Rokni M, Wistoft-Ibsen M. Optimization of hydrogen vehicle refueling via dynamic simulation. Int J Hydrogen Energy 2013;38:4221-4231.

[24] Rothuizen E, Rokni M. Optimization of the overall energy consumption in cascade fueling stations for hydrogen vehicles. Int J Hydrogen Energy 2014;98:582-592.

[25] Rothuizen E. Hydrogen fueling stations - A Thermodynamic analysis of fueling hydrogen vehicles for personal transportation. DTU Mechaninal Engeneering 2014. ISBN: 978-87-7475371-1.

[26] Society of Automotive Engineers. Fuelling protocols for light duty gaseous hydrogen surface vehicle. Technical Information Report J2601; 2010. https : //doi.org/10.4271/J2601_201003.

[27] Society of Automotive Engineers. Fuelling protocols for light duty gaseous hydrogen surface vehicle. Standard J2601; 2014. https : //doi.org/10.4271/J2601_201407.

[28] Society of Automotive Engineers. Fuelling protocols for light duty gaseous hydrogen surface vehicle. Standard J2601; 2016. https : //doi.org/10.4271/J2601_201612.

[29] Kawano Y,Kuroki T, Sakoda N, Monde M,Takata Y. Thermal analysis of high-pressure hydrogen during the discharging process. Int J Hydrogen Energy 2019;44:27039-27045.

[30] Kuroki T, Sakoda N, Shinzato K,Monde M,Takata Y. Dynamic simulation for optimal hydrogen refueling method to Fuel Cell Vehicle tanks. Int J Hydrogen Energy 2018;43:5714-5721.

[31] Schäfer S, Klein H. Thermodynamical analysis of a hydrogen fueling station via dynamic simulation. Int J Hydrogen Energy 2018;44:18240-18254

[32] Smith R. Chemical Process. 1 ed. New York John Wiley \& Sons; 2005, p. 273-275.

[33] Stridnig M, Brandstätter S, Sartory M,Klell M. Thermodynamic real gas analysis of a tank fillingprocess. Int J Hydrogen Energy 2014;39:8495-8509.

[34] Woodfield P, Monde M, Takano T. Heat transfer characteris- tics for practical hydrogen pressure vessels being filled at high pressure. J Therm Sci Tech-JPN 2008;3:214-253.

[35] Woodfield P, Monde M, Mitsutake Y. Measurement of averaged heat transfer coefficient in high-pressure vessel during charging with hydrogen nitrogen and argon gas. J Therm Sci Tech-JPN 2007:2:180-191.

[36] Yang J. A thermodynamic analysis of refueling of a hydrogen tank. Int J Hydrogen Energy 2009;34:6712-6721.

[37] Zhao L, Liu Y, Yang J, Zhao Y, Zheng J, Bie H, et al. Numerical simulation of temperature rise within hydrogen vehicle cylinder during refueling. Int J Hydrogen Energy 2010;35:8092-8100.

[38] Rothuizen E. Hydrogen fueling library. 2019. https : //github.com/DTU-TES/Hydrogen-fuelling-Station. 2.0, The 25th of November 2019. 